\title{
Don't Make a Habit Out of It: Impaired Learning Conditions Can Make Goal-Directed Behavior Seem Habitual
}

\author{
Eike Kofi Buabang ${ }^{1,2}$, Yannick Boddez ${ }^{3}$, Jan De Houwer ${ }^{3}$, Agnes Moors ${ }^{1,2}$
}

1. Research Group of Quantitative Psychology and Individual Differences, KU Leuven, Leuven, Belgium

2. Center for Social and Cultural Psychology, KU Leuven, Leuven, Belgium

3. Department of Experimental Clinical and Health Psychology, Ghent University, Ghent, Belgium

In press. Motivation Science.

Preregistration, materials, data, and analysis files can be found on the Open Science Framework: https://osf.io/xmup7/?view_only=2d1eaed5618f44f0967096a12f723f66 


\begin{abstract}
Habitual processes are often seen as the mechanisms underlying various suboptimal behaviors. Moors, Boddez, and De Houwer (2017) challenged this view, arguing that the influence of goaldirected processes may be underestimated in explaining suboptimal behavior. Much evidence for habitual processes in humans comes from studies that used an outcome devaluation test within a task called the Fabulous Fruit Game (FFG; de Wit, Niry, Wariyar, Aitken, \& Dickinson, 2007). In particular, poor performance on the FFG has been taken as evidence for increased reliance on habits. Recently, however, it was shown that the outcome devaluation test in the FFG targets the wrong outcome, which likely leads to an overestimation of habitual processes (De Houwer, Tanaka, Moors, \& Tibboel, 2018). We propose, in addition, that previous findings of differences in performance on the FFG do not reflect differences in habitual and goal-directed processing, but rather depend on differences in learning conditions such as task difficulty, and the opportunity, capacity, and motivation to learn the relevant contingencies. Our study shows that a lack of motivation leads to a pattern that would usually be interpreted as evidence for habits when in fact the behavior is goal-directed.
\end{abstract}

Keywords: goal-directed, habit, action slips, outcome devaluation test, motivation 


\section{Don't Make a Habit Out of It: Impaired Learning Conditions Can Make Goal-Directed Behavior Seem Habitual}

Suboptimal behavior is often seen as the result of an impaired balance between goaldirected and habitual processes. For example, it has been suggested that overreliance on habits is an underlying mechanism in cocaine addiction and obsessive-compulsive disorder (Ersche et al., 2016; Gillan et al., 2011). Goal-directed and habitual processes differ in the content of the mental representations involved. The mental representation in a goal-directed process consists of the contingency between responses and their outcomes (i.e., expectancy) given a certain stimulus and the value of the outcomes $\left(\mathrm{S}: \mathrm{R}-\mathrm{O}^{\mathrm{v}}\right)$. The mental representation in a habitual process consists of a stimulus-response link $(\mathrm{S}-\mathrm{R})$ and does not contain information about expectancies or values (Heyes \& Dickinson, 1990).

One way to diagnose whether a behavior is caused by a goal-directed or habitual process is through an outcome devaluation method, initially studied in rats (Dickinson, 1985). After an acquisition phase in which animals learn that the presence of a stimulus (S; e.g., auditory signal) followed by a response (R; e.g., pressing a lever) leads to a valued outcome $\left(\mathrm{O}^{\mathrm{v}}\right.$; e.g., food pellets), the outcome is devalued (e.g., through taste aversion). Finally, the stimulus is again presented, and it is tested whether responding decreases or remains the same. A decrease in responding is taken as evidence that the value of the outcome was represented and adjusted, indicating that the behavior was governed by a goal-directed process. The lack of such a devaluation effect (a status quo in responding) is interpreted as evidence that the value of the outcome was not represented, indicating that the behavior resulted from a habitual process.

In humans, one frequently used task that implements a devaluation test is the "Fabulous Fruit Game" (FFG; de Wit, Niry, Wariyar, Aitken, \& Dickinson, 2007; Gillan et al., 2011). 
During the acquisition phase, participants see a box that has a picture of a fruit on the outside (i.e., stimulus) and they have to respond by pressing a left or a right key (i.e., response). A correct response leads to an open box with a picture of a fruit inside (i.e., outcome) and points. An incorrect response leads to an empty box and no points. In one version of the $\mathrm{FFG}^{1}$, the devaluation and test phases are organized in blocks, with each block consisting of a devaluation phase and a test phase (de Wit et al., 2012). In each devaluation phase, participants see six boxes with fruit outcomes inside them, of which two are crossed out indicating that they no longer yield points. In each test phase, participants perform a go/no-go task. On each trial, a box with a fruit on the outside (i.e., stimulus) is presented and participants have to respond as quickly as possible if the fruit on the inside associated with it (i.e., outcome) is still valued and withhold from responding if it is devalued. Responses to stimuli that lead to devalued outcomes, so-called slips of action, are taken as evidence that they were caused by a habitual process.

Crucially, the acquisition phase comprises three trial types. On congruent trials, the stimulus fruit is the same as the outcome fruit that delivers points (e.g., a pear followed by a right-key response leads to a pear and points). On biconditional trials, the stimulus fruit is different from the outcome fruit (e.g., an apple followed by a left-key response leads to a melon and points). On incongruent trials, the stimulus fruit is different from the outcome fruit as well, but one fruit serves as the stimulus in some trials and as the outcome in other trials. When a fruit is presented as a stimulus, a different response is required than when it is presented as an

\footnotetext{
${ }^{1}$ In another version of the FFG, the outcome devaluation and the test phases are combined on trial level, and participants have to choose between a response that leads to a valued outcome and a response that leads to a devalued outcome on each trial (see de Wit et al., 2007). We used the go/no-go version of the FFG because it is the most frequently used and because participants have to respond under time pressure, which should increase the chance to observe action slips (Watson, van Wingen, \& de Wit, 2018).
} 
outcome (e.g., a cherry followed by a right-key response leads to grapes and points; grapes followed by a left-key response leads to a cherry and points).

De Wit et al. (2012) observed intact performance during acquisition on all three trial types but more action slips on incongruent than on other trial types during the test phase. Because on incongruent trials, the opposite response is required when a fruit is presented as a stimulus than when it is presented as an outcome, the same fruit is associated with two different responses (e.g., cherry as a stimulus is associated with a right-key response, cherry as an outcome with a left-key response) and therefore creates conflict. De Wit et al. (2007) hypothesized that participants solve this conflict by ignoring the outcome on incongruent trials, which comes down to the formation of a habitual link (i.e., via a $S-R$ link, e.g., cherry —rightkey response). Test performance is worse on the incongruent than on the other trial types because this performance relies on knowledge about the fruit outcomes, which is absent in the incongruent trials.

There are, however, reasons to believe that evidence for habits obtained with the FFG is overestimated. The presence of a devaluation effect supports the conclusion that behavior relies on a goal-directed process. Yet the absence of such an effect (even if combined with independent evidence of a devaluation effect in a control condition) is not necessarily due to a habit. If a behavior is not sensitive to the devaluation of one outcome, which indicates that it is not guided by this outcome, it may still be guided by other outcomes (Dickinson, 2016; Moors, Boddez, \& De Houwer, 2017). De Houwer, Tanaka, Moors, and Tibboel (2018) applied this argument to the FFG, in which responses are not only followed by fruit outcomes, but also by point outcomes. Therefore, insensitivity to the devaluation of the fruit outcomes, which indicates that it is not guided by these outcomes, does not rule out that behavior was still guided by the point outcomes. 
De Houwer et al. (2018) showed that participants were able to flexibly adjust responding after point outcome devaluation not only on congruent and biconditional trials but also on incongruent trials. This supports the idea that responding on incongruent trials is also goal directed, be it directed at the goal of obtaining point outcomes.

Even if the results of De Houwer et al. (2018) suggest that performance in the acquisition phase in all trial types can be explained in a goal-directed way, it remains an open question why de Wit et al. (2007, 2012) found that responding in the congruent and biconditional trials was sensitive to the fruit outcome devaluation whereas responding in the incongruent trials was not. De Houwer et al.'s (2018) studies suggest that during acquisition, intact performance on congruent and biconditional trials relied on a goal-directed process connecting stimuli, responses, fruit outcomes, and point outcomes ( $\mathrm{S}: \mathrm{R}$ - fruit $\mathrm{O}$ - point $\mathrm{O})$, whereas intact performance during incongruent trials relied on a goal-directed process connecting stimuli, responses, and point outcomes ( $\mathrm{S}: \mathrm{R}$ - point $\mathrm{O})$. Test performance is worse on incongruent trials because knowledge about the fruit outcomes is absent in these trials.

But why would fruit outcome knowledge not be learned on the incongruent trials despite instructions to do so? A straightforward explanation is that the extent to which participants learn about the fruit outcomes depends on general conditions for learning, such as task difficulty, opportunity, capacity, and motivation. Assuming that the sum of these learning conditions has to reach a certain threshold before learning occurs, we can further hypothesize that if the sum of these conditions is lowered, learning is impaired. Learning about the fruit outcomes is more difficult in the incongruent (because two fruits must be learned in addition to their roles as stimulus or outcome) than in the biconditional (in which only two fruits must be learned) and the congruent trials (in which only one fruit must be learned). Thus, it may be that the sum of the 
learning conditions in the incongruent trials was sufficient for participants to learn in a goaldirected way about the point outcomes, but not to also learn about the fruit outcomes. Given that successful learning about the fruit outcomes is measured by successful performance during the test phase, impaired performance during the test phase can be due to poor learning conditions during acquisition.

In addition to the role of task difficulty (de Wit et al., 2007), previous research already provides indications that learning about the fruit outcomes in the FFG depends on opportunity (e.g., de Wit, Ridderinkhof, Fletcher, \& Dickinson, 2013, found that increasing the number of acquisition trials led to equal performance on incongruent and biconditional trials) and capacity (e.g., Sjoerds et al., 2016, found positive correlations between test performance and visual shortterm memory). The current study will focus on the role of motivation to learn about the fruit outcomes as a determinant of learning. The motivation to learn something refers to the decision to engage in a set of mental actions ${ }^{2}(\mathrm{R})$, which should depend on the value of achieving the learning outcome (i.e., knowledge, O) and the expectancy ${ }^{3}$ that learning will lead to this learning outcome $(\mathrm{R}-\mathrm{O})$. Learning about the fruit outcomes is not required because the fruit outcomes are not valuable in themselves and only become so because they lead to points. Moreover, a crucial feature of the FFG is that learning about the fruit outcomes is not necessary to perform well during the acquisition (e.g., it is sufficient to know that cherry followed by a left-key response leads to points to correctly press the left key).

\footnotetext{
${ }^{2} \mathrm{We}$ are focusing here on the mental processes underlying learning (which was defined as an effect in De Houwer, Barnes-Holmes, \& Moors, 2013).

${ }^{3}$ Expectancy refers to subjective capacity, which is the extent to which an individual estimates that the set of mental actions required for learning will lead to successful learning. This may differ from the objective capacity an individual has to reach that outcome.
} 
Here, we examined the role of the motivation to learn about the fruit outcomes more directly by manipulating the goal relevance of the identity of the fruit outcomes. We did this by creating a variant of the FFG with congruent, biconditional, and incongruent trials, in which acquisition trials were intermixed with occasional probe trials. These are trials on which participants responded as usual, but instead of seeing the outcome, they received a question. In the high motivation group, the probe trials asked participants which fruit outcome they expected, and they were explicitly instructed to learn the identity of the fruit outcomes. In the low motivation group, the probe trials asked participants whether they expected any fruit outcome (without asking about the identity of the fruit outcome) and they were not instructed to learn the identity of the fruit outcomes. For both groups, no feedback was given about the responses to the probe trials to avoid creating additional opportunities to learn. We expected that this manipulation would be effective based on previous research, which showed that participants pick up contingencies in a learning task in a dimension that they are instructed to attend to (e.g., Eitam, Schul, \& Hassin, 2009). After acquisition, half of the participants in each motivation group were exposed to a devaluation of the fruit outcomes followed by a go/no-go task that relied on fruit outcome knowledge (i.e., the fruit devaluation condition). The other half of the participants in each motivation group were exposed to a devaluation procedure that was achieved via the reversal of the response-point outcome contingencies followed by a test that relied on point outcome knowledge (i.e., the point reversal condition).

Our first hypothesis was that low motivation to learn about the fruit outcomes would only impair performance (i.e., more slips of action) in the fruit devaluation condition (Hypothesis 1a) but not in the point reversal condition (Hypothesis $1 \mathrm{~b}$ ). Such a finding would suggest that both 
groups primarily learn in a goal-directed way based on the point outcomes $(\mathrm{S}: \mathrm{R}$ - point $\mathrm{O})$ and that learning about the fruit outcomes depends on the motivation to do so.

Our second hypothesis was that low motivation would particularly impair performance on incongruent trials. As suggested by the results of De Houwer et al. (2018), participants learn incongruent trials based on the point outcomes. Considering that learning about the fruit outcomes is more difficult for incongruent trials than on the other trial types, these trials may be most sensitive to the motivation manipulation because high motivation may help to overcome the difficulty. Thus, we expected that low motivation would impair performance for incongruent test trials compared to high motivation in the fruit devaluation condition (Hypothesis 2a). We did not expect differences between the two motivation groups for incongruent test trials in the point reversal condition (Hypothesis $2 b$ ).

Following previous work (De Houwer et al., 2018), we also assessed participants' explicit knowledge about the relations between responses and fruit outcomes ( $\mathrm{R}$ - fruit $\mathrm{O}$ knowledge) and between stimuli and fruit outcomes ( $\mathrm{S}$ - fruit $\mathrm{O}$ knowledge) as an additional measure of knowledge about the fruit outcomes. Worse explicit knowledge about the fruit outcomes has previously been taken as evidence for impaired goal-directed processing and overreliance on habits (Gillan et al., 2011). On our account, worse explicit knowledge does not indicate overreliance on habits, but simply that knowledge is worse as a result of poor learning conditions. In line with this, our third hypothesis was that the low motivation group would have worse knowledge about the relation between responses and fruit outcomes (Hypothesis 3a) and about the relation between stimuli and fruit outcomes (Hypothesis $3 b$ ) than the high motivation group. Finally, we tested the hypotheses that knowledge about the relations between responses and fruit outcomes (Hypothesis 4a) and about the relations between stimuli and fruit outcomes 
(Hypothesis 4b) would be associated with action slips in the fruit devaluation condition. This again would suggest that action slips are the result of less knowledge about the fruit outcomes. The first two hypotheses, sample size, and the procedure were preregistered with AsPredicted ${ }^{4}$. Although we did not preregister the third and fourth hypotheses, they are in line with those of previous research (De Houwer et al., 2018; Gillan et al., 2011).

We wish to point out that responding to a devalued fruit outcome during the test phase of the fruit devaluation condition can refer to two types of mistakes: incorrect responses that were correct during the acquisition phase and incorrect responses that were never correct. Although only the former type can be properly called action slips, both types of mistakes have not always been clearly separated in previous research. In our study, we will focus on proper slips of action only because this is the type of mistake that allows us to contrast our account with the habitual account.

\section{Method}

\section{Participants}

Two hundred participants (50 per condition, 170 female, 30 male, Age $M=20.50, S D=$ 3.82) were recruited from the participant pool of the Psychology Department at KU Leuven and completed the experiment for 4 Euros or in exchange for partial course credits. We conducted sensitivity analyses to determine the minimum detectable effect sizes given this sample size. The results of these analyses are reported in the results section.

\footnotetext{
${ }^{4}$ We preregistered separate analyses for each test type (fruit devaluation: mixed model ANOVA; point reversal: a comparison against chance level performance as in De Houwer et al., 2018). Ultimately, we deemed it more appropriate to analyze both test types in one analysis as reported here. The sample size was calculated based on the preregistered analyses. The sample size rationale and results of these analyses can be found at https://osf.io/xmup7/?view_only=2d1eaed5618f44f0967096a12f723f66.
} 
All participants provided informed consent and the study was approved by the KU Leuven ethical committee. Participants were tested in person and completed the study in individual cubicles.

\section{Procedure}

We created a variant of the FFG in which we manipulated trial type (congruent vs. biconditional vs. incongruent) within subjects, and motivation (high motivation vs. low motivation) and type of test (fruit vs. point) between subjects. The experiment consisted of an acquisition phase, a devaluation/reversal phase, and a test phase. In the acquisition phase, participants were assigned to either the high or low motivation group. In the devaluation/reversal phase, they were assigned to either a devaluation of the fruit outcomes or to a response-point outcome contingency reversal. The test phase consisted of trials in which participants were quickly presented with a stimulus and asked to respond in line with the devaluation/reversal. To familiarize themselves with the task, participants first practiced all three phases (using different pictures than those used in the experimental part). At the end of the experiment, participants' explicit knowledge about the relations between responses and fruit outcomes and between stimuli and fruit outcomes was assessed.

Acquisition phase. Stimuli and outcomes were randomly selected at the beginning of the acquisition phase from a pool of eight fruit pictures (apple, banana, cherry, grapes, kiwi, lemon, orange, pear, pineapple, strawberry). On each trial, participants saw a box with a picture on it (Figure 1a). They were asked to respond with a left ("D”-key) or right ("K"-key) keypress. If they responded correctly, they saw another picture of a fruit inside the box and a message indicating the number of points won on that trial (e.g., " +5 "). If they responded incorrectly, the box was empty and they did not receive any points. The box with the fruit on it was presented 
until participants responded, but faster responses were rewarded with more points. Responses faster than $1 \mathrm{~s}$ were rewarded with 5 points. For every additional $500 \mathrm{~ms}$, the points were reduced by one. Correct responses slower than $2.5 \mathrm{~s}$ were rewarded with one point. A counter above the box kept track of the point total. The opened box was presented for $1.5 \mathrm{~s}$. Then, the box disappeared and a blank screen was presented for $1.5 \mathrm{~s}$ before the next trial began. Participants were encouraged to earn as many points as possible.

The phase comprised three trial types (Figure 1b): congruent, biconditional, and incongruent. There were two stimuli for each trial type of which one was associated with the right-key response and the other with a left-key response. On congruent trials, the fruit on the box was the same as the fruit inside the box. On biconditional trials, the fruit on the box was different from the fruit inside the box. On incongruent trials, the fruit that was on the box in one contingency was inside the box in the other contingency. Ninety-six trials were presented divided over eight blocks. In each block each of the six stimuli was presented twice (16 times in total).

To manipulate motivation, we added probe trials (Figure 1c). These trials occurred after every six trials starting after the second block. Thus, there were 12 probe trials in total, two for each stimulus. On these trials, participants responded as usual. However, instead of receiving feedback about what is inside the box, they were asked a question depending on the group they were in. In the high motivation group, participants were asked which fruit they expected to be inside the box and had to type in their answer. In the low motivation condition, participants were asked if they expected any fruit inside the box and had to type in yes or no. Participants in the high but not the low motivation group were also instructed before the start of the acquisition phase to try to learn the fruits inside the boxes. 
Practice of the acquisition phase was done without probe trials. The pictures on and inside the box were randomly selected from a pool of three pictures (cup of coffee, glass of beer, glass of wine). Participants only practiced the congruent and biconditional trial types with one stimulus in each trial type.

Devaluation/reversal and test phases. In the fruit devaluation condition, participants completed six blocks in which a devaluation phase preceded a test phase (Figure 2). During the devaluation phase of each block, participants were presented for 20s with six open boxes with the outcomes from the acquisition phase inside. Two of these outcomes had a red cross superimposed on them, which indicated that they were devalued during that block and would lead to a deduction of one point. In each block, there was one devalued outcome per left and right key response and devalued outcomes were not of the same trial type. Each outcome was devalued in two blocks. During the test phase of each block, participants performed a go/no-go task. On each trial, they were presented with a closed box with a stimulus on it. The box was presented for $2 \mathrm{~s}$ and they had to quickly respond as before if the outcome (that had followed it during the practice acquisition phase after a correct response) was still valuable and withhold their response if the outcome was no longer valuable. In this phase, no feedback was given; participants saw a blank screen for $1.5 \mathrm{~s}$ after each response. In total, participants in the fruit devaluation condition completed 144 trials divided over six blocks (24 trials after each devaluation phase). Because each outcome was devalued in two blocks and each stimulus was presented four times per block, each stimulus was associated with a devalued outcome on 8 trials. Responses to a now devalued outcome that were correct in the acquisition phase were counted as action slips 
In the point reversal condition, participants completed one block in which the test was preceded by the instruction that responses that previously led to points would now lead to a deduction of one point, and responses that had not previously led to points would now lead to points. In the point reversal condition, each stimulus was presented 8 times to match the 8 times that each stimulus was presented when the outcome was devalued in the fruit devaluation condition. Thus, in total there were 48 trials. Responses that were correct in the acquisition phase were counted as action slips.

Practice of the devaluation/reversal and test phases was consistent with the condition that participants were assigned to. Participants assigned to the fruit devaluation condition received two practice blocks. In the devaluation phase of each block, two open boxes with the outcomes from the practice acquisition phase inside were presented with a red cross superimposed on one of the outcomes, indicating devaluation. The test phase consisted of six go/no-go trials and each stimulus was presented three times per block. Participant assigned to the point reversal condition received one practice block. After receiving the reversal instructions in the devaluation phase, they practiced eight trials in the test phase, and each stimulus was presented four times.

Explicit knowledge test. To test explicit knowledge about the relation between responses and fruit outcomes ( $\mathrm{R}$ - fruit $\mathrm{O}$ ), participants were presented on each trial with an open box with a fruit outcome inside and asked which key they needed to press to obtain that fruit. To test explicit knowledge about the relation between stimuli and fruit outcomes ( $\mathrm{S}$ - fruit $\mathrm{O}$ ), participants were presented on each trial with a picture of a fruit outcome inside the box, as well as all six pictures of fruit stimuli that were on the box and they were asked which of the fruit pictures was on the box given that the presented fruit was inside the box. In both explicit knowledge tests, the fruit outcomes were presented in random order. 


\section{Results}

The analyses were conducted using the R software (R Core Team, 2019) with the package rstatix (Kassambara, 2020). The first three hypotheses were analyzed using mixed model ANOVAs ${ }^{5}$. For each ANOVA that was conducted, the sphericity assumption was checked with Mauchly's test of sphericity. Corrected results (Greenhouse-Geisser correction) are reported when the sphericity assumption was violated. Significant effects of each ANOVA were followed up with pairwise comparisons, which were corrected using a Bonferroni adjustment for multiple testing. We conducted sensitivity analyses for the first hypothesis (two-way interaction between motivation and test type) and second hypothesis (three-way interaction between trial type, motivation, and test type) using the MorePower software (Campbell \& Thompson, 2012) to determine the minimum detectable effect sizes with a power of .80 given our sample size. Descriptive statistics for all analyses can be found in the supplementary materials on the Open Science Framework.

\section{Acquisition phase}

We conducted a mixed model ANOVA of the proportion of correct responses with block (1 to 8 ) and trial type (congruent, biconditional, incongruent) as within-subjects factors, and motivation (high vs. low) and test type (fruit vs. point) as between-subjects factors.

Results indicated a significant main effect of block, $F(5.22,1023.29)=166.78, p<.001$, $\eta^{2} p=.46$, suggesting that accuracy improved over blocks (Figure 3). We also found a significant main effect of motivation, $F(1,196)=6.45, p=.012, \eta^{2}{ }^{2}=.032$, with higher accuracy in the low

\footnotetext{
${ }^{5}$ We ran mixed model ANOVAs following previous analyses of the FFG (Gillan et al., 2011, de Wit et al., 2012). We note that the distribution of the residuals obtained with these models deviated from normality. However, it has been shown that these models are quite robust against normality deviations (Knief \& Forstmeier, 2020). Nevertheless, we also tested our hypotheses using nonparametrical factorial analyses using the ARTool package (Wobbrock, Findlater, Gergle, \& Higgins, 2011). The results of these alternative analyses can found here: https://osf.io/xmup7/?view_only=2d1eaed5618f44f0967096a12f723f66
} 
motivation group $(M=.85, S D=.23)$ than in the high motivation group $(M=.80, S D=.25)$. Although this result seems counterintuitive at first, a plausible interpretation is that the low motivation group primarily learned to respond correctly based on the point outcomes, whereas the high motivation group additionally focused on learning the fruit outcomes which is more complex.

There was also a significant main effect of trial type, $F(1.84,359.77)=51.22, p<.001$, $\eta_{p}^{2}=.21$, suggesting differences in performance between trial types. Pairwise comparisons with a corrected $\alpha=.017$ indicated that accuracy was significantly lower on incongruent trials $(M=$ $.77, S D=.26)$ than on congruent $(M=.86, S D=.22), t(1599)=-13.3, p<.001, d=-.33$, and biconditional trials $M=.85, S D=.23), t(1599)=-11.2, p<.001, d=-.28$, but did not differ between the latter two trials types, $t(1599)=2.24, p=.025, d=.06$. This pattern of effects is in line with previous studies (e.g., de Wit et al., 2007).

Results further indicated a four-way interaction between block, trial type, motivation, and test type, $F(11.19,2193.18)=1.96, p=.027, \eta^{2} p=.01$, which suggests further differences between groups over time, but is difficult to interpret. However, given this interaction and the differences between the two motivation groups, we decided to examine differences in accuracy by the end of the acquisition phase. For this purpose, we conducted an ANOVA of the proportion of correct responses in the last block. Results did not indicate differences between motivation groups, $F(1,196)=3.53, p=.062$, nor between test types, $F(1,196)=0.002, p=.964$, but the main effect of trial type persisted in the final block of acquisition, $F(2,392)=15.47, p<.001$, $\eta_{p}^{2}=.073$. Pairwise comparisons with a corrected $\alpha=.017$ indicated that accuracy was significantly lower on incongruent trials $(M=.87, S D=.19)$, than on congruent $(M=.95, S D=$ $.13), t(199)=-5.45, p<.001, d=-.39$, and biconditional trials $(M=.92, S D=.19), t(199)=-$ 
$3.16, p=.002, d=-.22$ but again no difference between the latter two trials types, $t(199)=2.38$, $p=.018, d=-.17$.

\section{Test phase}

We conducted a mixed model ANOVA of the proportion of action slips with trial type (congruent, biconditional, incongruent) as within-subjects factor, and motivation (high vs. low) and test type (fruit vs. point) as between-subjects factors. Action slips split by each factor are presented in Figure 4.

Our first hypothesis was that the low motivation group would make more action slips than the high motivation group in the fruit devaluation condition (1a), but that the proportion of action slips would not differ between the two motivation groups in the point reversal condition (1b). In line with our hypothesis, the interaction between motivation and test type was significant, $F(1,196)=5.05, p=.026, \eta^{2} p=.03$. A sensitivity analysis showed that, given the sample size, the minimum effect size detectable with a power of .80 is $\eta^{2} p=.04$. Thus, the observed effect size is slightly smaller than could be reliably detected given our sample size. Pairwise comparisons with a corrected $\alpha=.025$ were conducted to follow up the significant interaction. A one-tailed pairwise comparison confirmed that the low motivation group $(M=.32$, $S D=.34)$ made more slips of action than the high motivation group $(M=.21, S D=.28)$ in the fruit devaluation condition, $t(298)=3.09, p=.001, d=.36$. A two-tailed pairwise comparison did not yield significant differences in action slips between both groups in the point reversal condition (low motivation: $M=.17, S D=.25$; high motivation: $M=.21, S D=.25), t(298)=-$ $1.428, p=.203, d=-.15$. This absence of significant differences is in line with our hypothesis, keeping in mind that non-significant tests cannot be interpreted as evidence for equivalence, but as evidence for "trivial" differences at best if effect sizes are small (Friston, 2012). 
Our second hypothesis was that more action slips in the low compared to the high motivation group in the fruit devaluation condition would especially be found in for the incongruent trial type (2a). In the point reversal condition, we did not expect such a difference between the two motivation groups for the incongruent trials (2b). In line with our hypothesis, the three-way interaction between trial type, motivation, and test type was significant, $F(1.89$, $369.94)=5.08, p=.008, \eta^{2} p=.03$. A sensitivity analysis showed that, given the sample size, the minimum effect size detectable with a power of .80 is $\eta^{2} p=.02$. Thus, our sample size was sufficient to reliably detect the effect that we observed. Pairwise comparisons with a corrected $\alpha$ $=.008$ were conducted to follow up the significant interaction. One-tailed pairwise comparisons indicated that in the fruit devaluation condition, the low motivation group made more slips of action than the high motivation group on incongruent trials $\left(M_{\text {low }}=.44, S D=.39 ; M_{h i g h}=.24, S D\right.$ $=.31), t(98)=2.82, p=.003, d=.56$, but that slips of action did not differ between the low and high motivation groups on congruent trials $\left(M_{\text {low }}=.14, S D=.18 ; M_{\text {high }}=.13, S D=.18\right), t(98)=$ $0.27, p=.392, d=.05$, and biconditional trials $\left(M_{l o w}=.38, S D=.33 ; M_{\text {high }}=.26, S D=.32\right)$, $t(98)=1.90, p=.030, d=.38$. Two-tailed pairwise comparisons for the point reversal condition showed that slips of action did not differ between the low and high motivation groups on congruent trials $\left(M_{\text {low }}=.16, S D=.25 ; M_{\text {high }}=.18, S D=.22 ;\right), t(98)=-0.30, p=.763, d=-.06$, biconditional trials $\left(M_{\text {low }}=.18, S D=.26 ; M_{\text {high }}=.23, S D=.27\right), t(98)=-0.97, p=.336, d=-$ .19 , or incongruent trials $\left(M_{\text {low }}=.18, S D=.24 ; M_{\text {high }}=.22, S D=.24\right), t(98)=-0.89, p=.374, d$ $=-.17$. This absence of significant differences is in line with our hypothesis (again keeping in mind that this is not evidence of the lack of an effect, but evidence that any effect would not be more than trivial, Friston, 2012).

\section{Explicit knowledge test}


Our third hypothesis was that the low motivation group would have worse explicit knowledge than the high motivation group about relations between responses and fruit outcomes (3a) and about relations between stimuli and fruit outcomes (3b).

$\mathbf{R}-$ fruit $\mathbf{O}$ knowledge. We conducted a mixed model ANOVA of the proportion of correct responses with trial type (congruent, biconditional, incongruent) as within-subjects factor, and motivation (high vs. low) and test type (fruit vs. point) as between-subjects factors. Correct responses split by each factor are presented in Figure 5.

Contrary to Hypothesis 3a, there was no significant main effect of motivation, $F(1,196)$ $=1.14, p=.287$. Results indicated a main effect of trial type, $F(1.83,358.79)=27.20, p<.001$, $\eta_{p}^{2}=.12$. Pairwise comparisons with a corrected $\alpha=.017$ indicated that knowledge about the relation between responses and fruit outcomes was significantly better on congruent trials $(M=$ $.90, S D=.26)$ than on biconditional trials $(M=.80, S D=.34), t(199)=4.03, p>.001 d=.29$, and than on incongruent trials $(M=.68, S D=.42), t(199)=6.64, p<.001, d=.47$. This knowledge was also better on biconditional trials than on incongruent trials, $t(199)=3.78, p<$ $.001, d=.26$. The interaction between trial type and motivation was significant, $F(1.83,358.79)$ $=3.47, p=.036 . \eta^{2} p=.017$. Pairwise comparisons with a corrected $\alpha=.017$ were conducted to follow up the significant interaction. One-tailed pairwise comparisons indicated that the low motivation group $\left(M_{\text {low }}=.74, S D=.37\right)$ had worse knowledge on biconditional trials than the high motivation group $\left(M_{\text {high }}=.86, S D=.31\right), t(198)=-2.50, p=.007, d=-.35$. No significant differences in knowledge were found on congruent $\left(M_{\text {low }}=.91, S D=.24 ; M_{\text {high }}=.88, S D=.28\right)$, $t(198)=0.82, p=.207, d=.12$ and incongruent trials $\left(M_{\text {low }}=.67, S D=.42 ; M_{\text {high }}=.70, S D=\right.$ $.43), t(198)=-0.42, p=.338, d=-.06$. 
S-fruit O knowledge. We conducted an ANOVA of the proportion of correct responses on the test with trial type (congruent, biconditional, incongruent) as within-subjects factor, and motivation group (high vs. low) and test type (fruit vs. point) as between-subjects factors. Correct responses split by each factor are presented in Figure 6.

In line with Hypothesis $3 \mathrm{~b}$, there was a significant main effect of motivation, $F(1,196)=$ 23.96, $p<.001, \eta^{2} p=.109$, indicating that the low motivation group $(M=.74, S D=.38)$ had worse knowledge about the relation between stimuli and fruit outcomes than the high motivation group $(M=.92, S D=.24)$. There was also a significant interaction between trial type and motivation group, $F(2,392)=4.36, p=.013 \cdot \eta^{2}=.022$. One-tailed pairwise comparisons with a corrected $\alpha=.017$ indicated worse knowledge for the low than the high motivation groups on congruent trials $\left(M_{\text {low }}=.81, S D=.34 ; M_{\text {high }}=.91, S D=.27\right), t(198)=-2.42, p=.008, d=-.34$, on biconditional trials $\left(M_{\text {low }}=.72, S D=.36 ; M_{\text {high }}=.90, S D=.25\right), t(198)=-4.21, p<.001, d=$ .60 , and incongruent trials $\left(M_{\text {low }}=.70, S D=0.42 ; M_{\text {high }}=.935, S D=.21\right), t(198)=-5.12, p<$ $.001 d=.72$.

Our fourth hypothesis was that better knowledge of relations between responses and fruit outcomes (4a) and of relations between stimuli and fruit outcomes (4b) would be associated with less action slips in the fruit devaluation condition. As shown in Table 1, results were in line with our predictions, as better knowledge of both types of relations for each trial type was significantly negatively correlated with action slips for the respective trial type. In other words, the better the explicit knowledge for a trial type, the better the test performance for this trial type.

\section{Discussion}

The FFG has often been used to investigate the role of goal-directed and habitual processes in humans. It has been proposed that successful acquisition performance on the 
incongruent trials of the FFG is due to the formation of habits. This conclusion was based on results showing that participants have reduced knowledge about the fruit outcomes and make more action slips for this trial type during a subsequent test phase. We proposed that the extent to which participants learn about the fruit outcomes in the FFG depends on general conditions for learning, such as task difficulty, opportunity, capacity, and motivation. The present study examined the role of motivation, whereas previous studies already provided indications for the role of the other factors.

The primary finding of our study is that performance after fruit outcome devaluation depended on the motivation to learn about the fruit outcomes whereas performance after point outcome reversal was unaffected. Motivation was especially important for incongruent trials. These findings are in line with our account that during acquisition, participants give priority to learning about the relation between responses and point outcomes ( $\mathrm{S}: \mathrm{R}$-point $\mathrm{O})$. Participants can also learn about the fruit outcomes ( $\mathrm{S}: \mathrm{R}$ — fruit $\mathrm{O}$ - point $\mathrm{O}$ ) but, as we show, doing so depends on motivation. Therefore, even if there are differences in learning about the fruit outcomes, learning is still directed at the goal of obtaining points.

The second finding concerns the performance on the explicit knowledge tests, which was partially in line with our predictions: We did not find overall differences in explicit knowledge between the low and high motivation groups for relations between responses and fruit outcomes ( $\mathrm{R}$ - fruit O knowledge), but the low motivation group had worse knowledge for biconditional trials. For relations between stimuli and fruit outcomes ( $\mathrm{S}$ - fruit $\mathrm{O}$ knowledge) overall knowledge was worse for the low motivation group for each trial type. Further, we found that overall better knowledge of relations between responses and fruit outcomes and stimuli and fruit outcomes for each trial type was significantly associated with less action slips. This highlights 
that if motivation to learn about the fruit outcomes is not high enough, performance based on the fruit outcomes will inevitably be impaired.

The FFG has not only been used to study differences between trial types but also to compare different groups. Previous research has shown that compared to control groups, action slips (e.g., in biconditional trials) are more frequent in individuals with obsessive-compulsive disorder (OCD; Gillan et al., 2011), cocaine-dependence (Ersche et al., 2016), alcoholdependence (Sjoerds et al., 2013), sleep-deprivation (Chen et al., 2017), and stress (Fournier, D’Arripe- Longueville, \& Radel, 2017; Smeets, van Ruitenbeek, Hartogsveld, \& Quadflieg, 2018). The typical explanation is that individuals belonging to these groups rely more on habitual processes. Our study, however, suggests an alternative, goal-directed explanation for the observed differences in action slips. A number of previous findings could be attributed to a general deficit in motivation. If a task requires participants to learn several pieces of information, a general deficit in motivation may only allow them to learn some of it (e.g., only information about the point outcomes but not about the fruit outcomes). This is relevant because several clinical populations have been shown to exert less effort in instrumental behavior tasks (Barch, Pagliaccio, Luking, Moran, \& Culbreth, 2019; Salamone, Yohn, López-Cruz, San Miguel, \& Correa, 2016). Also, under certain conditions, such as sleep deprivation, effort is reduced (EngleFriedman, 2014). It is possible that differences in motivation may have contributed to studies of these groups with the FFG.

In the introduction, we already hinted at prior evidence for the role of other learning conditions for learning of the fruit outcomes besides motivation such as the opportunity and capacity to learn. Opportunity in the FFG can be linked to the number of trials in the acquisition phase. If the three trial types indeed differ in difficulty, more opportunity to learn should 
especially benefit learning of fruit outcomes on incongruent trials, which are the most difficult. Several studies show that increasing the original number of 8 trials per stimulus in the acquisition phase of the study by de Wit et al. (2007) leads to less slips of action on incongruent trials. de Wit, Ridderinkhof, Fletcher, and Dickinson (2013) found that extensive learning (144 trials per stimulus) leads to equal performance on incongruent and biconditional trials. Even after moderate training (36 trials per stimulus), performance was above chance level for incongruent trials. If one assumes that action slips are an index of habitual learning, one would have to accept the implausible conclusion that more training leads to weaker habits. The results of de Wit et al. (2013), however, make perfect sense if one assumes that the learning of relations between responses and fruit outcomes is more difficult on incongruent trials and thus requires more trials. Evidence for the influence of the capacity to learn about the fruit outcomes in the FFG has been delivered in the form of positive correlations between performance on the slips-ofaction test and scores on general intelligence and visual short-term memory measures (Sjoerds et al., 2016). Likewise, Boddez, Buabang, Zenses, and Descheemaeker (2018) invoked the lack of capacity as an alternative explanation for Chen et al.'s (2017) findings that sleep-deprived participants showed no difference with controls in performance during the acquisition phase, but a worse performance during the test phase. Thus, the capacity to learn may also influence whether or not participants can learn about the fruit outcomes.

Our proposal that actions slips after devaluation of the fruit outcomes depend on general conditions for learning provides an alternative explanation for previous mixed findings with the FFG in different populations. Whereas the studies that we listed earlier found more action slips in various populations compared to control groups (OCD; Gillan et al., 2011; cocainedependence, Ersche et al., 2016; alcohol-dependence, Sjoerds et al., 2013; sleep deprivation, 
Chen et al., 2017; stress, Fournier et al., 2017), this was not the case for people with autism spectrum disorder (Geurts \& de Wit, 2014), anorexia, (Godier et al., 2016), and obesity (Dietrich, de Wit, \& Horstmann, 2016). Some of the mixed results could be explained by differences in task difficulty, opportunity, capacity, and motivation, and the interaction between them. For instance, Gillan et al.'s (2011) OCD study had three trial types and each stimulus was presented 12 times during acquisition, whereas the acquisition phase in Geurts and de Wit's (2014) autism study had only one trial type and each stimulus was presented 20 times. Thus, the relatively higher task difficulty and lower opportunity in the OCD study, in combination with cognitive deficits in OCD patients (Jang et al., 2010) could explain the different findings.

It is also worth noting that learning conditions are not only relevant for learning about the fruit outcomes, but also for learning about the point outcomes. The congruency effect that we and others observed during the acquisition phase (de Wit et al., 2007), for instance, indicates that learning about the point outcomes is not equal between trial types. Similarly, some studies found that acquisition performance is not equal between groups. For example, in the study by Ersche et al. (2016), cocaine addicts had lower acquisition performance than the control participants persisting to the last block. If differences in learning between trials types and groups occurs already during the acquisition phase, impaired performance after devaluation is not surprising. Some of the limitations regarding the validity of the FFG as a method to study habitual processes have been acknowledged by Watson and de Wit (2018). They stated that slips of action could be the result of either increased reliance on habitual processes or reduced reliance on goaldirected processes, and they suggested that performance depends on a balance between the two processes. However, our study shows that performance may not even partly rely on habitual processes, because even if participants make action slips after the outcome devaluation in the 
fruit devaluation condition, they still performed well in the point reversal condition, which indicates that they did learn the relation between their responses and the point outcomes and hence that these responses were goal-directed.

A possible limitation of our study that could be raised is that the devaluation manipulation in the fruit devaluation condition was more complex than that in the point reversal condition. The fruit devaluation condition required remembering in each block which two (out of six) fruit outcomes were devalued whereas the point reversal condition merely required a response reversal (for each stimulus, give the opposite response of the one learned before). At first sight, this seems confirmed by the finding that participants in the fruit devaluation condition committed overall more action slips than those in the point reversal condition. Importantly, however, difficulty of the devaluation task cannot explain the interaction between type of devaluation and motivation. Only participants in the fruit devaluation condition who were also in the low motivation condition performed worse than participants in all other conditions. This suggests that our findings were due to motivation rather than to the difficulty of the devaluation task.

Another possible limitation is that participants practiced the acquisition phase and the test phase that they were assigned to at the beginning of the experiment. It is possible that the motivation to learn about the fruit outcomes was higher for participants who were assigned to the fruit devaluation condition compared to those who were assigned to the point reversal condition, as they knew that they would need this knowledge during a later phase of the experiment. However, the results of the acquisition phase and the explicit fruit knowledge tests do not suggest that mere practice gave an additional motivational benefit. There were no interactions between test type and motivation group, which would be expected if test type had an influence 
beyond motivation alone. The high and low motivation groups performed equally well during acquisition, independent of which test they anticipated in the test phase. Similarly, explicit knowledge was the same between the motivation groups, independent of test type.

As pointed out by De Houwer et al. (2018), the absence of a devaluation effect in the FFG may not necessarily indicate reliance on habitual processes. We showed in the present study that differences in the size of devaluation effects may also not indicate differences in habits but rather the extent to which people focus on certain outcomes. While the focus of the present paper was the FFG, our results have implications for other studies investigating the role of goaldirected and habitual processes. For any paradigm, it is crucial that performance in the test phase probes for knowledge that was successfully acquired. This is particularly relevant for tasks in which the values of outcomes (e.g., fruit) are installed by linking them with other outcomes (e.g., points). In all such tasks, it is possible that performance during acquisition is driven by the other outcome and therefore that knowledge about the outcome that is devalued is never successfully acquired. One way to try to minimize this problem is by ensuring that the learning conditions allow for sufficient learning. This problem may also be avoided by using outcomes that have inherent value, such as in the paradigm developed by Valentin, Dickinson, and O'Doherty (2007), which uses food outcomes. However, even if it is ensured that the outcome is acquired properly, the absence of a devaluation effect may still not provide sufficient evidence for habits because behavior might be directed at another goal. Thus, our findings also contribute to the general idea that the role of habits may be overestimated in explaining suboptimal behavior (Moors et al., 2017).

The central point of our paper is that goal-directed behavior in the FFG may seem habitual if learning conditions are poor. Interestingly, a similar argument has been made for 
performance in the two-stage task, which is used to distinguish between model-based and modelfree control. For instance, Feher da Silva and Hare (2020) showed that behavior in the two-stage task is almost exclusively model-based but may seem model-free due to inaccurate models or poor instructions.

In conclusion, we illustrated within the context of the FFG the idea that the general conditions for learning such as task difficulty, opportunity, capacity, and motivation are the central explanatory factors for seemingly habitual behavior. The identification of these factors comes with the advantage that they provide clear targets for behavior change. As we showed in our study, increasing motivation significantly improved performance. Future research could examine how the different factors interact with each other. To summarize, our study supports the idea that differences in devaluation effects can result from differences in motivation to engage in the goal-directed learning of outcomes rather than a shifted balance between goal-directed and habitual processes involved in learning and/or deployment. 


\section{References}

Barch, D. M., Pagliaccio, D., Luking, K., Moran, E. K., \& Culbreth, A. J. (2019). Pathways to motivational impairments in psychopathology: Common versus unique elements across domains. Nebraska Symposium on Motivation, 121-160. https://doi.org/10.1007/978-3030-27473-3_5

Boddez, Y., Buabang, E. K., Zenses, A., \& Descheemaeker, M. (2018). Commentary: Sleep deprivation promotes habitual control over goal-directed control: Behavioral and Neuroimaging evidence. Frontiers in Behavioral Neuroscience, 12. https://doi.org/10.3389/fnbeh.2018.00082

Buabang, E. K., Boddez, Y., De Houwer, J., \& Moors, A. (2020, September 23). Don't Make a Habit Out of It: Impaired Learning Conditions Can Make Goal-Directed Behavior Seem Habitual. Retrieved from osf.io/xmup

Campbell, J. I., \& Thompson, V. A. (2012). MorePower 6.0 for ANOVA with relational confidence intervals and Bayesian analysis. Behavior Research Methods, 44, 1255-1265. doi:10.3758/s13428-012-0186-0

Chen, J., Liang, J., Lin, X., Zhang, Y., Zhang, Y., Lu, L., \& Shi, J. (2017). Sleep deprivation promotes habitual control over goal-directed control: Behavioral and neuroimaging evidence. The Journal of Neuroscience, 37, 11979-11992. https://doi.org/10.1523/jneurosci.1612-17.2017

De Houwer, J., Tanaka, A., Moors, A., \& Tibboel, H. (2018). Kicking the habit: Why evidence for habits in humans might be overestimated. Motivation Science, 4, 50-59. doi: $10.1037 / \operatorname{mot} 0000065$ 
De Houwer, J., Barnes-Holmes, D., \& Moors, A. (2013). What is learning? On the nature and merits of a functional definition of learning. Psychonomic Bulletin \& Review, 20, 631642. https://doi.org/10.3758/s13423-013-0386-3

de Wit, S., Niry, D., Wariyar, R., Aitken, M. R., \& Dickinson, A. (2007). Stimulus-outcome interactions during instrumental discrimination learning by rats and humans. Journal of Experimental Psychology: Animal Behavior Processes, 33, 1-11. doi:10.1037/00977403.33.1.1

de Wit, S., Ridderinkhof, K. R., Fletcher, P. C., \& Dickinson, A. (2013). Resolution of outcome-induced response conflict by humans after extended training. Psychological Research, 77, 780-793. doi:10.1007/s00426-012-0467-3

de Wit, S., Watson, P., Harsay, H. A., Cohen, M. X., Van de Vijver, I., \& Ridderinkhof, K. R. (2012). Corticostriatal connectivity underlies individual differences in the balance between habitual and goal-directed action control. Journal of Neuroscience, 32, 1206612075. doi:10.1523/jneurosci.1088-12.2012

Dickinson, A. (1985). Actions and habits: The development of behavioural autonomy. Philosophical Transactions of the Royal Society B: Biological Sciences, 308, 67-78. doi:10.1098/rstb.1985.0010

Dickinson, A. (2016). Instrumental conditioning revisited: Updating dual-process theory. In J. B. Trobalon \& V. D. Chamizo (Eds.), Associative learning and cognition: Homage to Professor NJ Mackintosh. In Memoriam (1935-2015), 177-195.

Dietrich, A., de Wit, S., \& Horstmann, A. (2016). General habit propensity relates to the sensation seeking subdomain of impulsivity but not obesity. Frontiers in Behavioral Neuroscience, 10. doi:10.3389/fnbeh.2016.00213 
Eitam, B., Schul, Y., \& Hassin, R. R. (2009). Short article: Goal relevance and artificial grammar learning. Quarterly Journal of Experimental Psychology, 62, 228238. https://doi.org/10.1080/17470210802479113

Engle-Friedman, M. (2014). The effects of sleep loss on capacity and effort. Sleep Science, 7, 213-224. https://doi.org/10.1016/j.slsci.2014.11.001

Ersche, K. D., Gillan, C. M., Jones, P. S., Williams, G. B., Ward, L. H., Luijten, M., ... Robbins, T. W. (2016). Carrots and sticks fail to change behavior in cocaine addiction. Science, 352, 1468-1471. doi:10.1126/science.aaf3700

Feher da Silva, C., \& Hare, T. A. (2020). Humans primarily use model-based inference in the two-stage task. Nature Human Behaviour. https://doi.org/10.1038/s41562-020-0905-y

Fournier, M., D’Arripe- Longueville, F., \& Radel, R. (2017). Effects of psychosocial stress on the goal-directed and habit memory systems during learning and later execution. Psychoneuroendocrinology, 77, 275-283.

doi:10.1016/j.psyneuen.2016.12.008

Friston, K. (2012). Ten ironic rules for non-statistical reviewers. NeuroImage, 61, 13001310. https://doi.org/10.1016/j.neuroimage.2012.04.018

Gillan, C. M., Papmeyer, M., Morein-Zamir, S., Sahakian, B. J., Fineberg, N. A., Robbins, T. W., \& De Wit, S. (2011). Disruption in the balance between goal-directed behavior and habit learning in obsessive-compulsive disorder. American Journal of Psychiatry, 168, 718-726. doi:10.1176/appi.ajp.2011.10071062

Geurts, H. M., \& de Wit, S. (2014). Goal-directed action control in children with autism spectrum disorders. Autism, 18, 409-418. doi:10.1177/1362361313477919 
Godier, L. R., de Wit, S., Pinto, A., Steinglass, J. E., Greene, A. L., Scaife, J., ... Park, R. J. (2016). An investigation of habit learning in Anorexia Nervosa. Psychiatry Research, 244, 214-222. doi:10.1016/j.psychres.2016.07.051

Heyes, C., \& Dickinson, A. (1990). The intentionality of animal action. Mind \& Language, 5, 87-103. https://doi.org/10.1111/j.1468-0017.1990.tb00154.x

Jang, J. H., Kim, H. S., Ha, T. H., Shin, N. Y., Kang, D. H., Choi, J. S., ... \& Kwon, J. S. (2010). Nonverbal memory and organizational dysfunctions are related with distinct symptom dimensions in obsessive-compulsive disorder. Psychiatry Research, 180, 93-98. doi:10.1016/j.psychres.2010.04.016.

Kassambara, A. (2020). rstatix: Pipe-Friendly Framework for Basic Statistical Tests. R package version 0.6.0. https://CRAN.R-project.org/package=rstatix

Moors, A., Boddez, Y., \& De Houwer, J. (2017). The power of goal-directed processes in the causation of emotional and other actions. Emotion Review, 9, 310-318. doi:10.1177/1754073916669595

R Core Team (2019). R: A language and environment for statistical computing. R Foundation for Statistical Computing, Vienna, Austria. URL http://www.R-project.org/

Salamone, J. D., Yohn, S. E., López-Cruz, L., San Miguel, N., \& Correa, M. (2016). Activational and effort-related aspects of motivation: Neural mechanisms and implications for psychopathology. Brain, 139, 1325-1347. https://doi.org/10.1093/brain/aww050

Sjoerds, Z., de Wit, S., Van den Brink, W., Robbins, T. W., Beekman, A. T., Penninx, B. W., \& Veltman, D. J. (2013). Behavioral and neuroimaging evidence for overreliance on habit 
learning in alcohol-dependent patients. Translational Psychiatry, 3, e337-e337. doi:10.1038/tp.2013.107

Sjoerds, Z., Dietrich, A., Deserno, L., de Wit, S., Villringer, A., Heinze, H., ... Horstmann, A. (2016). Slips of action and sequential decisions: A cross-validation study of tasks assessing habitual and goal-directed action control. Frontiers in Behavioral Neuroscience, 10. doi:10.3389/fnbeh.2016.00234

Smeets, T., van Ruitenbeek, P., Hartogsveld, B., \& Quaedflieg, C. W. (2018). Stress-induced reliance on habitual behavior is moderated by cortisol reactivity. Brain and Cognition. doi:10.1016/j.bandc.2018.05.005

Valentin, V. V., Dickinson, A., \& O'Doherty, J. P. (2007). Determining the neural substrates of goal-directed learning in the human brain. Journal of Neuroscience, 27, 40194026. https://doi.org/10.1523/jneurosci.0564-07.2007

Watson, P., \& de Wit, S. (2018). Current limits of experimental research into habits and future directions. Current Opinion in Behavioral Sciences, 20, 33-39.

doi:10.1016/j.cobeha.2017.09.012 
Table 1

Correlations between $R$ - fruit $O$ and $S$ - fruit $O$ Knowledge and Proportion of Action Slips in the Fruit Devaluation Condition per Trial Type

\begin{tabular}{lll}
\hline Trial Type & $\mathrm{R}-\mathrm{O}$ & $\mathrm{S}-\mathrm{O}$ \\
\hline Congruent & $-0.27 * *$ & $-0.47 * * *$ \\
Biconditional & $-0.35 * * *$ & $-0.57 * * *$ \\
Incongruent & $-0.47 * * *$ & $-0.61 * * *$ \\
& & \\
\hline Note. $* * p<.01, * * * p<.001$ &
\end{tabular}


(a) Acquisition trials

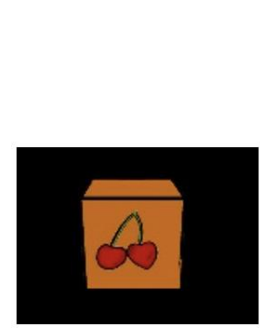

(b) Probe trials

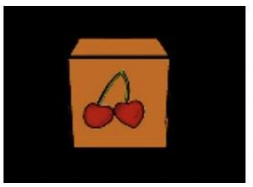

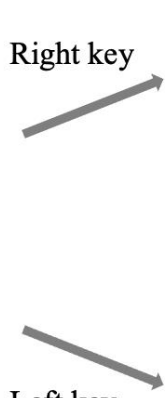

Left key

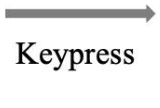

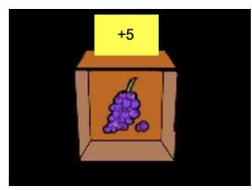

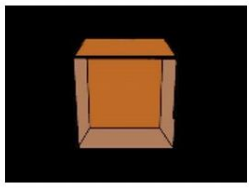

Low motivation

Do you expect a fruit

inside the box?

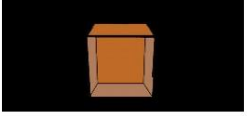

High motivation

Which fruit do you expect inside the box?

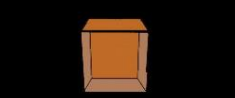

(c) Trial types

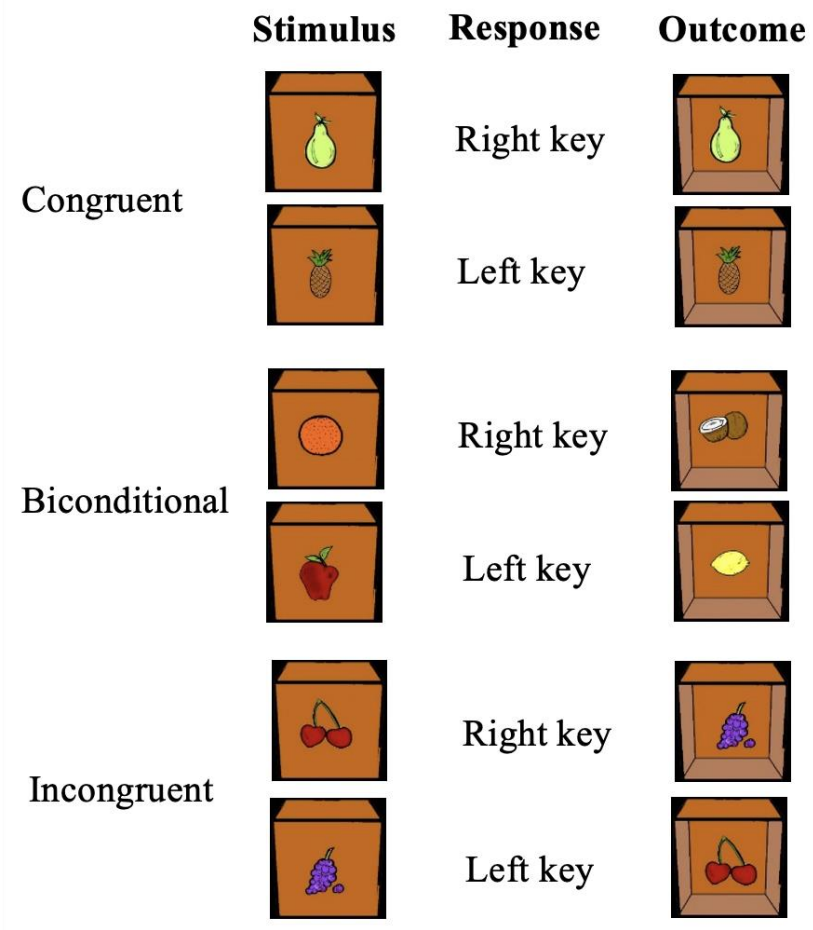

Figure 1. Structure of the acquisition phase. (a) On acquisition trials, participants were presented with a box with a fruit on the outside. Correct responses led to a opened box with a fruit on the inside and points. (b) On probe trials, participants received a question that differed per motivation group. (c) In total, the acquisition phase conisisted of 6 contingencies. 


\section{Fruit devaluation}

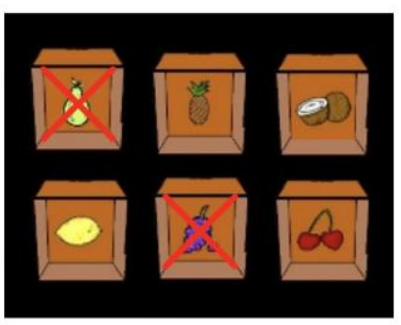

\section{Point reversal}

From now on, the key

presses that previously led

to points now lead to a

subtraction of one point,

and the key presses that

did not lead to points now

lead to points.

\section{Test}

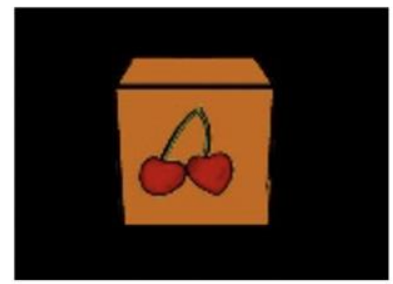

Figure 2. In the devaluation/reversal and test phases, participants either received a fruit outcome devaluation or a point contingency reversal. In the fruit devaluation condition, in each block two fruit outcomes had a red cross superimposed on them, which indicated that they were devalued during that block and would lead to a deduction of one point. In the point reversal condition, participants received an instructed $\mathrm{R}$ - point $\mathrm{O}$ contingency reversal. In the test phase, participants in both conditions were presented on each trial for $2 \mathrm{~ms}$ with the fruit stimuli from the acquisition phase and had to respond in line with the knowledge from the devaluation/reversal phase. 


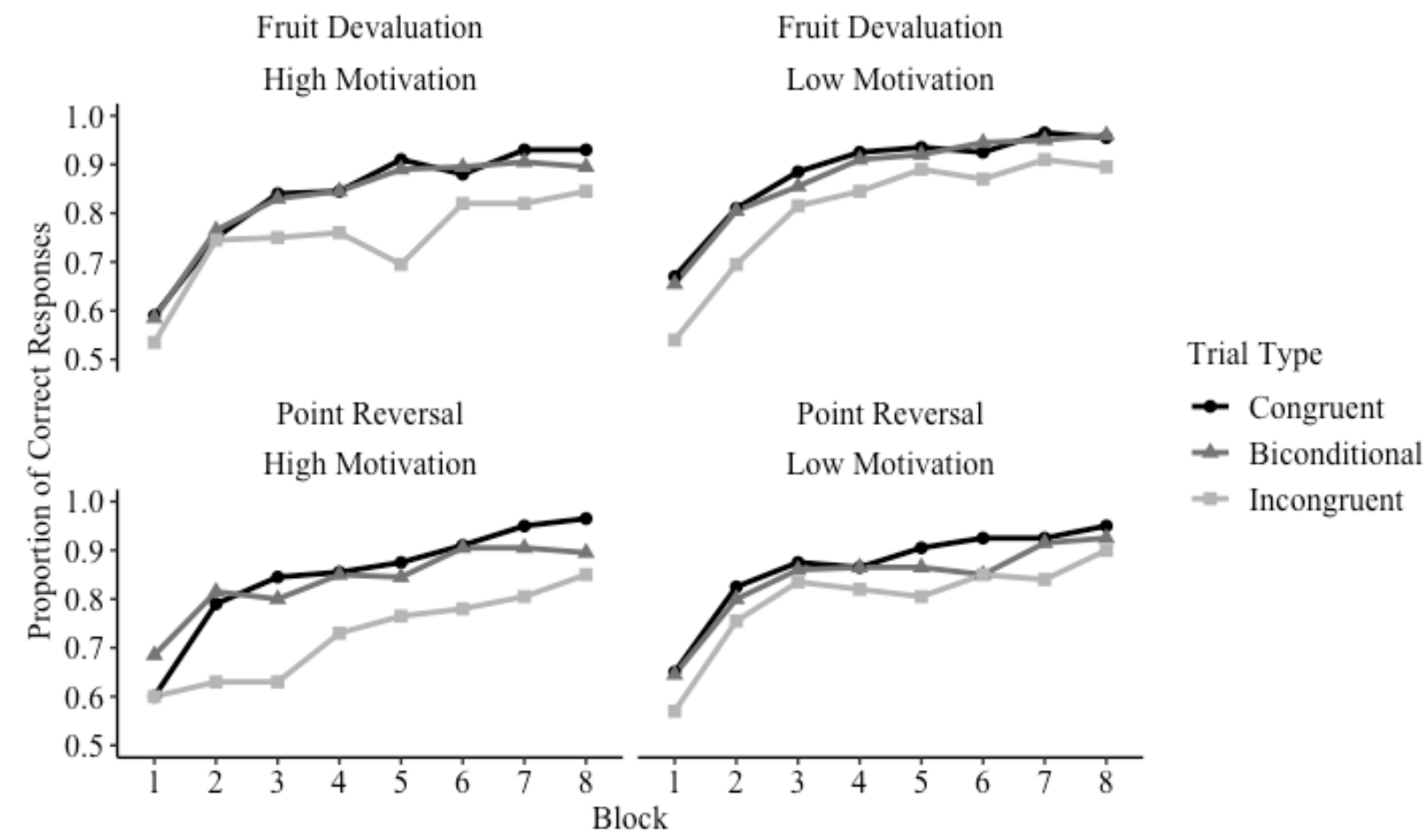

Figure 3. Proportion of correct responses during acquisition over blocks per devaluation condition, motivation group, and trial type. 


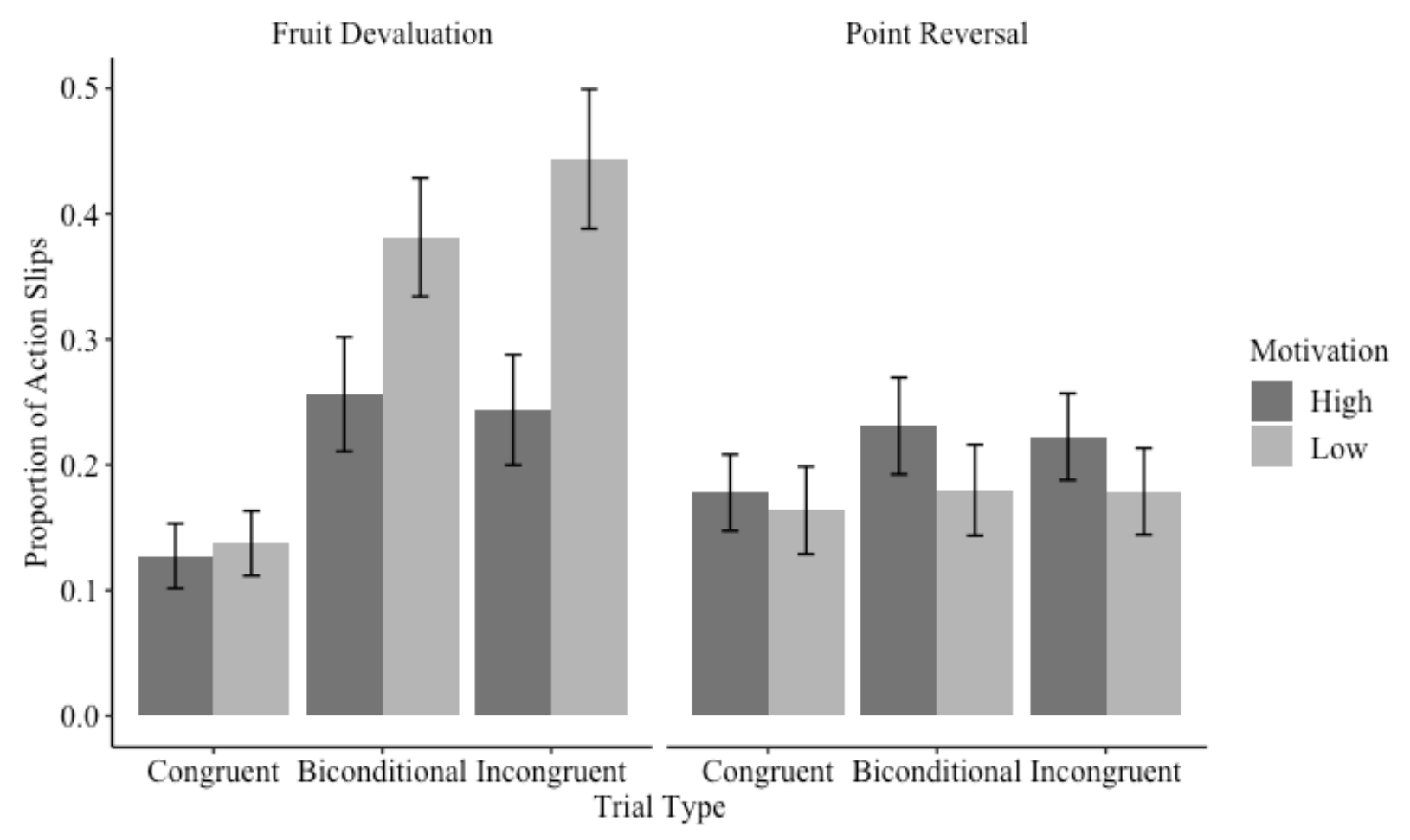

Figure 4. Proportion of action slips per devaluation condition, motivation group, and trial type. Error bars represent standard errors. 


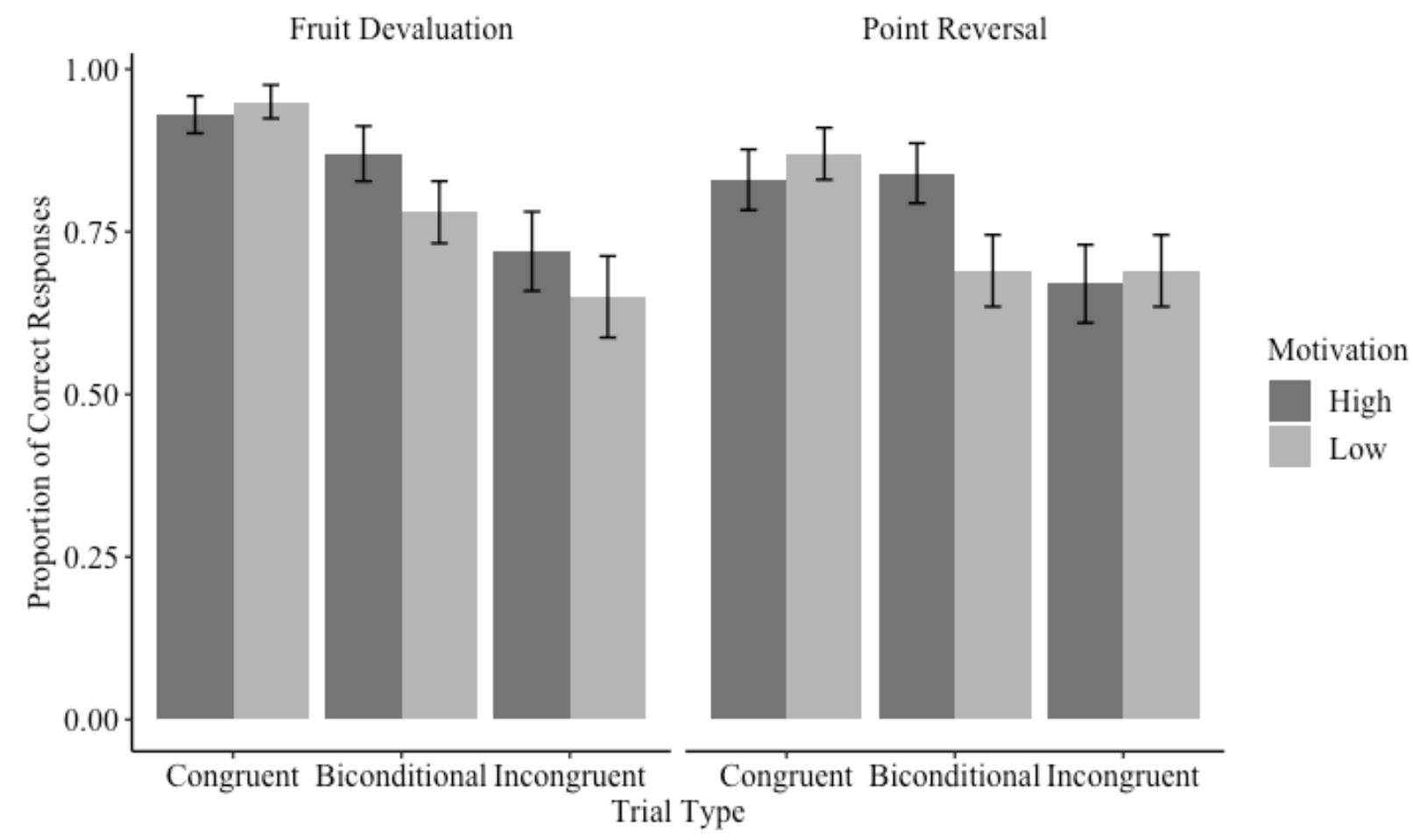

Figure 5. Correct responses in the R-O knowledge test per devaluation condition, motivation group, and trial type. Error bars represent standard errors. 


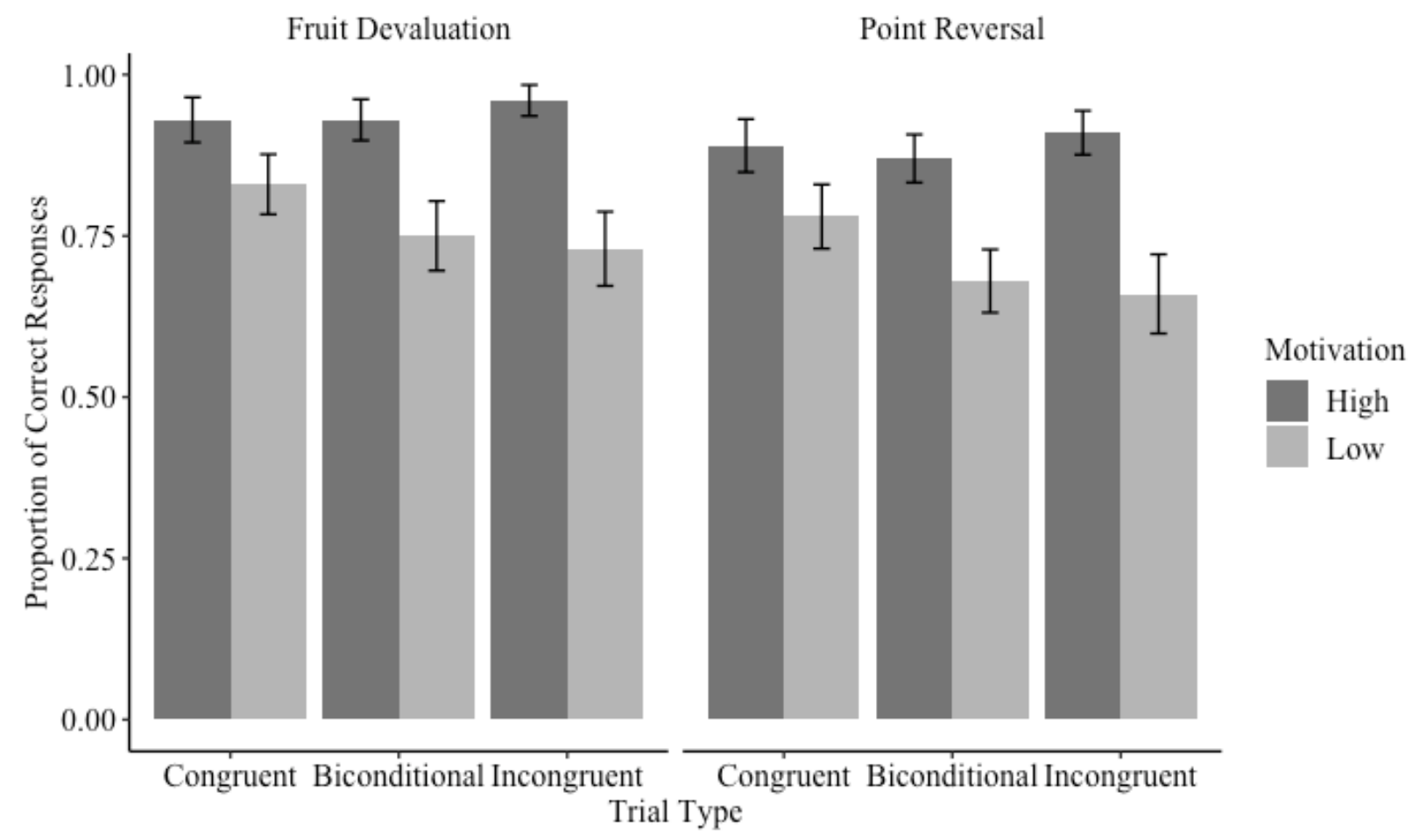

Figure 6. Correct responses in the S-O knowledge test per devaluation condition, motivation group, and trial type. Error bars represent standard errors. 